\title{
On the Convergence of Orbits in Sequence Space $\ell^{2}$
}

\author{
Marco Mpimbo \\ Department of Mathematics, University of Dar es Salaam, Tanzania. \\ Email: kmpimbo33@gmail.com
}

Received 23 Mar 2021, Revised 29 Jul 2021, Accepted 30 Jul 2021, Published Aug 2021

DOI: https://dx.doi.org/10.4314/tjs.v47i3.26

\begin{abstract}
This paper discusses the convergence of orbits for diagonal operators defined on $\ell^{2}$. In particular, the basis elements of $\ell^{2}$ are obtained using the linear combinations of the elements of the orbit. Furthermore, via the classical result of the determinant of the Vandermonde matrix, it is shown that, the more the elements of the orbit are used, the faster the convergence of the orbit to the basis elements of $\ell^{2}$.
\end{abstract}

Keywords: Diagonal operators, Convergence of Orbits of operators, Vandermonde matrix, Norm topology.

2010 Mathematics Subject Classification: 47A16.

\section{Introduction}

The theory of sequences is equally important both in Pure and Applied Mathematics. For instance, in Computer programming and Numerical Analysis, the solutions of some problems are dependent on whether the convergence is possible or not.

Let $\left\{L_{j}\right\}_{j=1}^{\infty}$ be a sequence of bounded linear operators from a Banach space $X$ to a normed linear space $Y$, and suppose that the sequence converges pointwise to a bounded linear operator $L$, that is, $L(x)=\lim L_{n}(x)$ for each $x \in X$. A natural question that arises here is: What can be said about the rate or speed of this convergence? This is an interesting and important question that has attracted many researchers over the decades. In this study, a particular case is considered: When the sequence $\{L\}_{j=1}^{\infty}$ is generated by the powers of a single linear operator $T$, that is, $L_{j}=T^{j}$ for each $j$. Other types of convergences that are closely related to pointwise convergence and that are related to the type of convergence discussed in this study are arbitrarily slow convergence and linearly(fast) convergence. The sequence $\left\{L_{n}\right\}_{n=1}^{\infty}$ is said to converge to $L$ arbitrarily slowly provided it converges pointwise, and for each sequence of real numbers $\{\phi(n)\}_{n=1}^{\infty}$ with $\lim _{n \rightarrow \infty} \phi(n)=0$, there exists $x=x_{\phi} \in X \quad$ such that $x=x_{\phi} \in$ $\left\|L_{n}(x)-L(x)\right\| \geq \phi(n)$ for each $n$, while it is said to be linear if there exist constants $\alpha \in$ $[0,1)$ and a real number $c$ such that $\| L_{n}-$ $L \| \leq c \alpha^{n}$ for each $n$.

Deutsch and Hundal (2010) studied the rate of convergence of a sequence of linear operators that converge pointwise to a linear operator. Their main result gives the useful conditions that guarantee arbitrarily slow convergence. In the particular case, when the sequence of linear operators is generated by the powers of a single linear operator, they state that: either there is linear (fast) convergence or arbitrarily slow convergence; no other type of convergence is possible. On the other hand, Bögli (2017) established the spectral convergence results of approximations of 
Mpimbo - On the Convergence of Orbits in Sequence Space $\ell^{2}$

unbounded non-self adjoint linear operators with compact resolvents by operators that converge in generalized strong resolvent sense. In particular, the general assumptions that ensure spectral exactness are established by using the discrete compactness of the sequence of resolvents of the approximating operators. Consequently, the sufficient conditions and perturbation results for strong convergence and for discrete compactness of the resolvents are formulated.

If $T$ is a continuous linear operator on a Banach space $X$ and $x \in X$, then the orbit of $x$ under $T$ is the sequence $\left(x, T x, T^{2} x, \ldots\right)$. We say that $x$ is cyclic if the closed, linear span of the orbit is the whole space; that is, if $\overline{\operatorname{span}}\left\{T^{j} x\right\}_{j=1}^{\infty}=X$. The study of orbits in connection with cyclic vectors has been for a while and some classical problems in analysis can be viewed as problems on orbits of operators. Given a bounded linear operator on a Banach space $X$, a (closed) subspace $Y$ of $X$ is called an invariant subspace for $T$ if $T(Y) \subset$ $Y$.

The classical invariant subspace problem explains whether every continuous linear operator T on a Banach space X has a closed non-trivial invariant subspace. This question can be reformulated in terms of orbits as follows: Is there an operator $T$ on $X$ such that every $x \neq 0$ is cyclic? For Banach spaces; the problem was solved by Enflo in 1975, but the paper with this result was published in 1987: Enflo (1987) constructed a Banach space $X$ and an operator $T$ on $X$ such that every $x \neq 0$ is cyclic. On the other hand, Read (1985) gave examples of operators with only trivial invariant subspaces on some of the classical Banach spaces, in particular $\ell^{1}$ and $c_{0}$.

An outstanding observation of usefulness of cyclic vectors is seen in Weierstrass's classical approximation theorem, which states that every continuous function on the interval $(0,1)$ can be approximated uniformly by polynomials $p(t)$. This result can be reformulated as a result of orbits of operators as follows: Let $C(0,1)$ be the space of continuous real-valued functions on $(0,1)$ with $\|f\|=\max _{t \in[0,1]}|f(t)|$. Let $L^{2}[0,1]$ denote the space of square-integrable functions on $[0,1]$ with $\|f\|=\left(\int_{0}^{1}|f(t)|^{2} d t\right)^{\frac{1}{2}}$. Let $T$ denote multiplication by $t$ in the space $C(0,1)$ (or $\left.L^{2}[0,1]\right), \quad$ i.e., $\quad(T f)(t)=$ $t f(t)$. Weierstrass's approximation theorem then becomes: The constant function $f(t) \equiv 1$ $i$ syclic for $T$ in the space $C(0,1)$. From this result, it is easily verified that, the constant function $f(t) \equiv 1$ is also cyclic in $L^{2}[0,1]$. A strengthening of Weierstrass's theorem was given by Szász (1916) who proved that not only is $f(t) \equiv 1$ cyclic, but also a sufficiently dense subsequence of the orbit of the constant function 1 spans the whole space; more precisely, $\quad \overline{\operatorname{span}}\left\{t^{n j}\right\}_{j=0}^{\infty} \cup\{1\}=$ $C[0,1]\left(\operatorname{or} L^{2}[0,1]\right)$ if and only if $\sum_{j=1}^{\infty} \frac{1}{n_{j}}=\infty$.

Mančevska and Orovčanec (2007) proved the following assertion: If $\left\{T_{n}\right\}_{n=1}^{\infty}$ is a sequence of bounded linear operators on infinite-dimensional Banach space $X$, where the spectral radius $r\left(T_{i}\right)>1$ for all $i$, then there exists a dense set of vectors in $X$ with orbits tending to infinity under every operator in the sequence. In connection to this, is the result due to Müller and Vršovský (2009) which states that if $T$ is a bounded linear operator on a Banach space $X$ satisfying the condition $\sum_{n=1}^{\infty} \frac{1}{\left\|T^{n} x\right\|}<\infty$, then there exists a unit vector $x \in X$ such that $\left\|T^{n} x\right\| \rightarrow \infty$. This result implies the existence of nontrivial closed invariant subspace under $T$.

However, in the invariant subspace (or subset) problem, the main focus is whether the vectors are cyclic or not. One does not consider more detailed questions about the orbits, for example, in what ways are the orbits cyclic?

In this paper, the linear combinations of the elements of the orbit are analyzed in detail and use this argument to determine how fast one can approach the basis elements of $\ell^{2}$.

\section{Materials and Methods}


This study is mainly theoretical, so the tools that were used are diagonal operators, eigenvalues, basis elements of $\ell^{2}$ and the Vandermonde matrix. Also, definitions and lemmas were used to establish the main results. The main method used throughout the paper is the method of computing norms in $\ell^{2}$. Also, the standard approximation techniques are used whenever necessary.

\section{Preliminaries and definitions}

- $T=\operatorname{diag}\left(\lambda_{1}, \lambda_{2}, \lambda_{3}, \ldots\right)$ denotes the diagonal operator,

$$
T=\left(\begin{array}{ccccc}
\lambda_{1} & 0 & 0 & \ldots & \\
0 & \lambda_{2} & 0 & \ldots & \\
0 & 0 & \lambda_{3} & 0 & \ldots \\
\vdots & \vdots & \vdots & \ddots & \vdots
\end{array}\right)
$$

relative to a sequence

of vectors $\left(e_{i}\right)$.

- $\quad\left(e_{i}\right)$ will be the natural basis of $\ell^{2}$.

- $\left\|x-x_{0}\right\| \rightarrow 0$ means the strong convergence of an element $x \in \ell^{2}$ to an element $x_{0} \in \ell^{2}$.

- Vandermonde matrix: An $n \times n$ Vandermonde matrix

$A=\left(\begin{array}{ccccc}1 & \alpha_{1} & \alpha_{1}{ }^{2} & \ldots & \alpha_{1}{ }^{n-1} \\ 1 & \alpha_{2} & \alpha_{2}{ }^{2} & \ldots & \alpha_{2}{ }^{n-1} \\ 1 & \alpha_{3} & \alpha_{3}{ }^{2} & \ldots & \alpha_{3}{ }^{n-1} \\ \vdots & \vdots & \vdots & & \vdots \\ 1 & \alpha_{n} & \alpha_{n}{ }^{2} & \ldots & \alpha_{n}{ }^{n-1}\end{array}\right) \quad$ is $\quad$ a matrix whose terms are a geometric progression in each row. Its determinant is computed by the formula,

$|A|=\prod_{1 \leq i \leq j \leq n}\left(\alpha_{j}-\alpha_{i}\right)$, and it is non-zero if and only all $\alpha_{i}$ are distinct.

Definition 1.1. If $S$ is a subspace of a Hilbert space $H$, then we define the orthogonal complement of $S$ by $S^{\perp}=\{f \in H:(f, g)=$ $0, \forall g \in S\}$.

Definition 1.2. Let $(X, d)$ be a metric space. We define $d(x, A)=i n f_{y \in A} d(x, y)$.
Lemma 1.3. Let $T: \ell^{2} \rightarrow \ell^{2}$ be a diagonal operator as defined above and let $\left(e_{j}\right)_{j=1}^{\infty}$ be the standard basis for $\ell^{2}$. If $S T=T S$, then $e_{j}$ is an eigenvector of $S$.

Proof. Since $T$ is a diagonal, we have that $T e_{j}=\lambda_{j} e_{j}, \forall j$. Since $S T=T S$, we have,

$$
\begin{gathered}
T S\left(e_{j}\right)=S T\left(e_{j}\right) \\
=S\left(T\left(e_{j}\right)\right) \\
=S\left(\lambda_{j} e_{j}\right) \\
=\lambda_{j} S\left(e_{j}\right)
\end{gathered}
$$

$\Rightarrow S\left(e_{j}\right)$ is an eigenvector of $T$ with an eigenvalue $\lambda_{j}$

$\Rightarrow S\left(e_{j}\right)=\gamma e_{j}$ for some scalar $\gamma$.

Thus, $e_{j}$ is an eigenvector of $S$.

Remark 1.4. The next three lemmas are classical results and can be found in any standard functional analysis textbooks, hence we skip their proofs.

Lemma 1.5. If $T: H \rightarrow H$ is self-adjoint and $F \subset H \quad$ (closed linear subspace of $H$ ) is invariant under $T$, then $F^{\perp}$ is also invariant under $T$.

- In this case we say that $F$ reduces $T$ or that $T$ is reduced by $F$.

Lemma 1.6. Let $\mathrm{P}$ be the orthogonal projection on a closed linear subspace $F$ of $H$. Then $F$ is invariant under an operator $T$ if and only if $T P=P T P$.

Lemma 1.7. Let $P_{F}$ be the orthogonal projection on a closed linear subspace $F \subset H$. Then $F \quad$ reduces $\quad T \Leftrightarrow T P_{F}=P_{F} T$ (i.e. $P_{F}$ commutes with $T$ ).

\section{Main Results}

The main results are started by first noting the following:

Remark 2.1. If a diagonal operator $T=$ $\operatorname{diag}\left(\lambda_{1}, \lambda_{2}, \lambda_{3}, \ldots\right)$, has $\lambda_{i}=\lambda_{j}$ for some $i \neq$ 
$j$, then it will not have any cyclic vector, since if $\lambda_{i}=\lambda_{j}, x=\left(x_{1}, x_{2}, \ldots, x_{i}, \ldots, x_{j}, \ldots\right)$, then

$T^{n} x=\left(\lambda_{1}^{n} x_{1}, \lambda_{2}^{n} x_{2}, \ldots, \lambda_{i}^{n} x_{i}, \ldots, \lambda_{j}^{n} x_{j}, \ldots\right)$ and so the ratio between the $i^{\text {th }}$ and $j^{\text {th }}$ coordinates of $T^{n} x$ is $\frac{x_{i}}{x_{j}}$ for all $n$. So we will only consider $T$ where $\lambda_{i} \neq \lambda_{j}$ if $i \neq j$ and $\lambda_{j}>0$ for all $j$. The first result shows that a vector , $x=\left(x_{1}, x_{2}, x_{3} \ldots\right) \in \ell^{2}$ is cyclic if and only if $x_{i} \neq 0$ for all $i$.

\section{Theorem 2.2}

Let $T=\left(\begin{array}{ccccc}\lambda_{1} & 0 & 0 & \ldots & \\ 0 & \lambda_{2} & 0 & \ldots & \\ 0 & 0 & \lambda_{3} & 0 & \ldots \\ \vdots & \vdots & \vdots & \ddots & \vdots\end{array}\right), \quad T: \ell^{2} \rightarrow \ell^{2}$ be a diagonal operator with distinct positive eigenvalues and $x \in \ell^{2}$. Then $x=$ $\left(x_{1}, x_{2}, x_{3} \ldots\right)$ is cyclic if and only if $x_{i} \neq 0$ for all $i$.

Proof. To prove the forward direction, we apply the lemmas above.

First; we write $H$ as $H=F \oplus F^{\perp}$, then we have that, $F$ reduces $T \Leftrightarrow T P_{F}=P_{F} T$ (by Lemma 1.7). Thus, $P_{F}$ has the same eigenvectors as $T$ (by Lemma 1.3). But then Lemma 1.5 says that $F^{\perp}$ is an invariant subspace of $H$. But we know that $T_{e_{j}}=\lambda_{j} e_{j} \forall j$ and,

(i) $P_{F}\left(e_{j}\right)=e_{j}$ whenever $e_{j} \in F$

(ii) $P_{F}\left(e_{j}\right)=0$ whenever $e_{j} \in F^{\perp}$

So, we have that for the orthogonal projection $P_{F}$, either $\lambda_{j}=1$ or $\lambda_{j}=0$ for all $j$. Thus, all eigenvectors of $T$ for which $P_{F}$ has the eigenvalue 1, they span $F$ and all eigenvectors of $T$ for which $P_{F}$ has the eigenvalue 0 , they span $F^{\perp}$. But $x_{j} \neq 0$ for all $j$, hence $x=$ $\left(x_{1}, x_{2}, x_{3} \ldots\right)$ must be cyclic.

For the converse, it is enough to note that, if $x_{i}=0$ for some $i_{0}$, then $T^{n} x_{i_{0}}=0$ for all $n$. Hence $T^{n} x$ does not span the whole space, therefore $x$ is not cyclic.

Remark 2.3. The above theorem gives the characterization of cyclic vectors in $\ell^{2}$, but it does not give any information on how vectors are cyclic, for example, what type of linear combinations of vectors in the orbit should be used to approximate a given vector in a space $\ell^{2}$. To answer this question, we restrict our study to a subclass of diagonal operators where the eigenvalues are considered positive and decreasing. In so doing, we capture the notation of convergence of orbits with respect to this subclass of diagonal operators.

First, consider a $T$ where $\lambda_{1}>\lambda_{2}>\lambda_{3} \ldots$ and all $\lambda_{i}$ 's are positive and consider a vector $x \in \ell^{2}$ where $x_{i} \neq 0$ for all $i$. Notice that, if $\lambda_{1}>\lambda_{2}>\lambda_{3} \ldots, T=\operatorname{diag}\left(\lambda_{1}, \lambda_{2}, \lambda_{3}, \ldots\right)$ a diagonal operator, and $x=\left(x_{1}, x_{2}, x_{3} \ldots\right)$ is a vector in $\ell^{2}$ such that $x_{1} \neq 0$. Then,

$$
\frac{T^{n} x}{\lambda_{1}^{n} x_{1}}=\frac{1}{\lambda_{1}^{n} x_{1}}\left(\lambda_{1}^{n} x_{1}, \lambda_{2}^{n} x_{2}, \lambda_{3}^{n} x_{3}, \ldots\right)=\left(1,\left(\frac{\lambda_{2}}{\lambda_{1}}\right)^{n} \frac{x_{2}}{x_{1}},\left(\frac{\lambda_{3}}{\lambda_{1}}\right)^{n} \frac{x_{3}}{x_{1}}, \ldots\right)
$$

Thus, $\frac{T^{n} x}{\lambda_{1}^{n} x_{1}} \rightarrow(1,0,0, \ldots)$ as $n \rightarrow \infty$; and for $n$ large enough we have,

Further, since

$$
\left\|\frac{T^{n} x}{\lambda_{1}^{n} x_{1}}-(1,0,0, . .)\right\| \leq 2\left(\frac{\lambda_{2}}{\lambda_{1}}\right)^{n}\left|\frac{x_{2}}{x_{1}}\right|
$$

We also get,

$$
\left\|T^{n} x\right\|^{2}=\left(\lambda_{1}^{n} x_{1}\right)^{2}\left(1+\left(\frac{\lambda_{2}}{\lambda_{1}}\right)^{2 n}\left(\frac{x_{2}}{x_{1}}\right)^{2}+\left(\frac{\lambda_{3}}{\lambda_{1}}\right)^{2 n}\left(\frac{x_{3}}{x_{1}}\right)^{2}+\cdots\right)
$$

for $n$ large enough.

$$
\left\|\frac{T^{n} x}{\left\|T^{n} x\right\|}-(1,0,0, \ldots)\right\| \leq 2\left(\frac{\lambda_{2}}{\lambda_{1}}\right)^{n}\left|\frac{x_{2}}{x_{1}}\right|
$$


Thus, the orbit converges. In order to study this further, we will focus on the diagonal operators with decreasing eigenvalues. In the next theorem we will study how much faster we can get convergence to $x=(1,0,0, \ldots)$ if we use larger linear combinations of the elements of the orbit.

$$
d\left((1,0,0, \ldots), \operatorname{span}\left\{T^{n} x, T^{(n-1)} x, \ldots, T^{(n-(k-1))} x\right\}\right) \leq C_{(k, x)}\left(\frac{\lambda_{k}}{\lambda_{1}}\right)^{n},
$$

where $C_{(k, x)}$ depends on $k$.

Proof. The idea of the proof is to construct a linear combination of the elements $\left\{T^{j} x\right\}_{j=n}^{n-(k-1)}$ to approximate the basis vectors $\left(e_{i}\right)$. It is enough to produce the first basis vector as similar technique can be employed to get the rest of the basis vectors. We start by giving the proofs for $k=2$ and $k=3$ and then pass to the general case.
Theorem 2.4. Let $T: \ell^{2} \rightarrow \ell^{2}, \quad T=$ $\operatorname{diag}\left(\lambda_{1}, \lambda_{2}, \lambda_{3}, \ldots\right)$ be an infinite diagonal operator with distinct eigenvalues decreasing to zero; that is, $\lambda_{1}>\lambda_{2}>\lambda_{3} \ldots \rightarrow 0$. Suppose $x=\left(x_{1}, x_{2}, x_{3} \ldots\right) \in \ell^{2}$ where $x_{i} \neq 0$ for all $i$. Then,
Case 1: We estimate (2.1) by using the first two elements, $T^{n} x$ and $T^{n-1} x$ of the orbit. Notice that, $T^{n} x=\left\{\lambda_{1}^{n} x_{1}, \lambda_{2}^{n} x_{2}, \lambda_{3}^{n} x_{3}, \ldots\right\}$ and

$$
T^{n-1} x=\left\{\lambda_{1}^{n} x_{1}, \lambda_{2}^{n} x_{2}, \lambda_{3}^{n} x_{3}, \ldots\right\} .
$$

Since $\lambda_{1}>\lambda_{2}>\lambda_{3} \ldots \rightarrow 0$, One has that, $\left\|T^{n} x\right\| \approx \lambda_{1}^{n} x_{1}$ and $\left\|T^{n-1} x\right\| \approx \lambda_{1}^{n-1} x_{1}$ for large enough $n$, where the symbol $\approx$ means that the ratio between the sides tends to 1 as $n$ tends to $\infty$. Now, let,

and

$$
Z_{1}=\frac{T^{n} x}{\lambda_{1}^{n} x_{1}}=\left(1,\left(\frac{\lambda_{2}}{\lambda_{1}}\right)^{n} \frac{x_{2}}{x_{1}},\left(\frac{\lambda_{3}}{\lambda_{1}}\right)^{n} \frac{x_{3}}{x_{1}}, \ldots\right)
$$

We show that,

$$
Z_{2}=\frac{T^{n-1} x}{\lambda_{1}^{n} x_{1}}=\left(1,\left(\frac{\lambda_{2}}{\lambda_{1}}\right)^{n-1} \frac{x_{2}}{x_{1}},\left(\frac{\lambda_{3}}{\lambda_{1}}\right)^{n-1} \frac{x_{3}}{x_{1}}, \ldots\right) .
$$

$$
d\left((1,0,0, \ldots), \operatorname{span}\left\{T^{n} x, T^{(n-1)} x\right\}\right) \leq C_{(3, x)}\left(\frac{\lambda_{3}}{\lambda_{1}}\right)^{n}
$$

Indeed, if we take $a_{1}=\left(\frac{\lambda_{2}}{\lambda_{1}}\right)^{n}$ and $a_{2}=\left(\frac{\lambda_{2}}{\lambda_{1}}\right)^{n-1}$, then by elementary computations we get,

For large enough $n$.

$$
\begin{aligned}
\frac{Z_{2}}{a_{2}}-\frac{Z_{1}}{a_{1}} & =\left(\left(\frac{\lambda_{2}}{\lambda_{1}}-1\right)\left(\frac{\lambda_{2}}{\lambda_{1}}\right)^{n} \frac{x_{2}}{x_{1}}, 0,\left(\frac{\lambda_{3}}{\lambda_{1}}-1\right)^{n} \frac{x_{3}}{x_{1}} \ldots\right) . \\
& \Rightarrow\left\|\frac{Z_{2}}{a_{2}}-\frac{Z_{1}}{a_{1}}\right\| \approx\left(\frac{\lambda_{2}}{\lambda_{1}}-1\right)\left(\frac{\lambda_{2}}{\lambda_{1}}\right)^{n} \frac{x_{2}}{x_{1}}
\end{aligned}
$$

By letting $Z_{1,2}=\frac{\frac{z_{2}}{a_{2}}-\frac{z_{1}}{a_{1}}}{\left\|\frac{z_{2}}{a_{2}}-\frac{z_{1}}{a_{1}}\right\|}$ we have that, for a suitable b

So,

$$
b \frac{Z_{1,2}}{\left\|Z_{1,2}\right\|}=\left(1,0, \frac{\left(\frac{\lambda_{2}}{\lambda_{3}}-1\right)}{\left(\frac{\lambda_{2}}{\lambda_{1}}-1\right)}\left(\frac{x_{3}}{x_{1}}\right)^{n} \frac{x_{3} x_{1}}{\left(x_{2}\right)^{2}}, \ldots\right) .
$$


Hence,

$$
d\left((1,0,0, \ldots), \operatorname{span}\left\{Z_{1}, Z_{2}\right\}\right) \approx \frac{\left(\frac{\lambda_{2}}{\lambda_{3}}-1\right) x_{3} x_{1}}{\left(\frac{\lambda_{2}}{\lambda_{1}}-1\right)\left(x_{2}\right)^{2}}\left(\frac{\lambda_{3}}{\lambda_{1}}\right)^{n}
$$

Where

$$
d\left((1,0,0, \ldots), \operatorname{span}\left\{T^{n} x, T^{(n-1)} x\right\}\right) \leq C_{(3, x)}\left(\frac{\lambda_{3}}{\lambda_{1}}\right)^{n}
$$

$$
C_{(3, x)}=\frac{\left(\frac{\lambda_{2}}{\lambda_{3}}-1\right) x_{3} x_{1}}{\left(\frac{\lambda_{2}}{\lambda_{1}}-1\right)\left(x_{2}\right)^{2}}
$$

Case 2: Now, we estimate (2.1) by using the first three elements of the orbit: $T^{n} x, T^{n-1} x$ and $T^{n-2} x$.

Let $Z_{1}, Z_{2}, a_{1}, a_{2}$ be defined as in step 1 above, $a_{3}=\left(\frac{\lambda_{2}}{\lambda_{1}}\right)^{n-2}$ and choose $b_{1}$ such that

We show that,

$$
z_{3}=b_{1} \frac{T^{n-2} x}{\left\|T^{n-2} x\right\|}=\left(1,\left(\frac{\lambda_{2}}{\lambda_{1}}\right)^{n-2} \frac{x_{2}}{x_{1}},\left(\frac{\lambda_{3}}{\lambda_{1}}\right)^{n-2} \frac{x_{3}}{x_{1}}, \ldots\right) .
$$

$$
d\left((1,0,0, \ldots), \operatorname{span}\left\{T^{n} x, T^{(n-1)} x, T^{n-2} x\right\}\right) \leq C_{(4, x)}\left(\frac{\lambda_{4}}{\lambda_{1}}\right)^{n}
$$

If we let $c=\frac{\lambda_{2}}{\lambda_{3}}+1$, then we have the following:

$$
\begin{aligned}
& \frac{Z_{3}}{a_{3}}-\frac{c Z_{2}}{a_{2}}+\frac{(c-1) Z_{1}}{a_{1}} \\
& =\left(\left(\frac{\lambda_{2} \lambda_{3}-\lambda_{1} \lambda_{2}}{\lambda_{1} \lambda_{3}}\right)\left(\frac{\lambda_{2}}{\lambda_{1}}-1\right)\left(\frac{\lambda_{1}}{\lambda_{2}}\right)^{n} \frac{x_{1}}{x_{2}}, 0,0,\left(\frac{\lambda_{2} \lambda_{3}-\lambda_{2} \lambda_{4}}{\lambda_{4} \lambda_{3}}\right)\left(\frac{\lambda_{2}}{\lambda_{4}}\right.\right. \\
& \left.-1)\left(\frac{\lambda_{4}}{\lambda_{2}}\right)^{n} \frac{x_{4}}{x_{2}}, \ldots\right)
\end{aligned}
$$

By letting

One has, for a suitable $b_{2}$,

$$
Z_{1,2,3}=\frac{Z_{3}}{a_{3}}-\frac{c Z_{2}}{a_{2}}+\frac{(c-1) Z_{1}}{a_{1}},
$$

$$
b_{2} \frac{Z_{1,2,3}}{\left\|Z_{1,2,3}\right\|}=\left((1,0,0,) \frac{\left(\frac{\lambda_{2} \lambda_{3}-\lambda_{2} \lambda_{4}}{\lambda_{4} \lambda_{3}}\right) x_{4}}{\left(\frac{\lambda_{2} \lambda_{3}-\lambda_{1} \lambda_{2}}{\lambda_{1} \lambda_{3}}\right) x_{1}}\left(\frac{\lambda_{4}}{\lambda_{1}}\right)^{n}, \ldots\right) .
$$

So,

Thus,

$$
d\left((1,0,0, \ldots), \operatorname{span}\left\{Z_{1}, Z_{2} Z_{3}\right\}\right) \approx \frac{\left(\frac{\lambda_{2} \lambda_{3}-\lambda_{2} \lambda_{4}}{\lambda_{4} \lambda_{3}}\right) x_{4}}{\left(\frac{\lambda_{2} \lambda_{3}-\lambda_{1} \lambda_{2}}{\lambda_{1} \lambda_{3}}\right) x_{1}}\left(\frac{\lambda_{4}}{\lambda_{1}}\right)^{n}
$$




$$
d\left((1,0,0, \ldots), \operatorname{span}\left\{T^{n} x, T^{(n-1)} x, T^{n-2} x\right\}\right) \approx \frac{\left(\frac{\lambda_{2} \lambda_{3}-\lambda_{2} \lambda_{4}}{\lambda_{4} \lambda_{3}}\right) x_{4}}{\left(\frac{\lambda_{2} \lambda_{3}-\lambda_{1} \lambda_{2}}{\lambda_{1} \lambda_{3}}\right) x_{1}}\left(\frac{\lambda_{4}}{\lambda_{1}}\right)^{n}
$$

Hence,

$$
d\left((1,0,0, \ldots), \operatorname{span}\left\{T^{n} x, T^{(n-1)} x, T^{n-2} x\right\}\right) \approx C_{(4, x)}\left(\frac{\lambda_{4}}{\lambda_{1}}\right)^{n} .
$$

Where

$$
C_{(4, x)}=\frac{\left(\frac{\lambda_{2} \lambda_{3}-\lambda_{2} \lambda_{4}}{\lambda_{4} \lambda_{3}}\right) x_{4}}{\left(\frac{\lambda_{2} \lambda_{3}-\lambda_{1} \lambda_{2}}{\lambda_{1} \lambda_{3}}\right) x_{1}}
$$

General Case: Careful examination of cases 1 and 2 above reveals that the proof of the theorem will be complete if we can take the linear combinations of $k$ elements of the orbit and produce a vector with first coordinate one, the rest zeros except the $k^{\text {th }}$ position, then

$$
\begin{gathered}
\alpha_{1} Z_{1}=\alpha_{1}\left(1,\left(\frac{\lambda_{2}}{\lambda_{1}}\right)^{n} \frac{x_{2}}{x_{1}},\left(\frac{\lambda_{3}}{\lambda_{1}}\right)^{n} \frac{x_{3}}{x_{1}}, \ldots\right) \\
\alpha_{2} Z_{2}=\alpha_{2}\left(1,\left(\frac{\lambda_{2}}{\lambda_{1}}\right)^{n-1} \frac{x_{2}}{x_{1}},\left(\frac{\lambda_{3}}{\lambda_{1}}\right)^{n-1} \frac{x_{3}}{x_{1}}, \ldots\right) \\
\alpha_{3} Z_{3}=\alpha_{3}\left(1,\left(\frac{\lambda_{2}}{\lambda_{1}}\right)^{n-2} \frac{x_{2}}{x_{1}},\left(\frac{\lambda_{3}}{\lambda_{1}}\right)^{n-2} \frac{x_{3}}{x_{1}}, \ldots\right) \\
=\vdots \\
\alpha_{k} Z_{k}=\alpha_{k}\left(1,\left(\frac{\lambda_{k}}{\lambda_{1}}\right)^{n-(k-1)} \frac{x_{2}}{x_{1}},\left(\frac{\lambda_{k}}{\lambda_{1}}\right)^{n-(k-1)} \frac{x_{3}}{x_{1}}, \ldots\right)
\end{gathered}
$$

But then, we have to have the following happen:

$$
\begin{gathered}
\alpha_{1}+\alpha_{2}+\alpha_{3}+\cdots+\alpha_{k}=1 \\
\alpha_{1}+\alpha_{2}\left(\frac{\lambda_{2}}{\lambda_{1}}\right)^{n-1} \frac{x_{2}}{x_{1}}+\cdots+\alpha_{k}\left(\frac{\lambda_{2}}{\lambda_{1}}\right)^{n-(k-1)} \frac{x_{2}}{x_{1}}=0 \\
\alpha_{1}\left(\frac{\lambda_{3}}{\lambda_{1}}\right)^{n} \frac{x_{2}}{x_{1}}+\alpha_{2}\left(\frac{\lambda_{3}}{\lambda_{1}}\right)^{n-1} \frac{x_{2}}{x_{1}}+\cdots+\alpha_{k}\left(\frac{\lambda_{3}}{\lambda_{1}}\right)^{n-(k-1)} \frac{x_{2}}{x_{1}}=0 \\
\alpha_{1}\left(\frac{\lambda_{k}}{\lambda_{1}}\right)^{n} \frac{x_{2}}{x_{1}}+\alpha_{2}\left(\frac{\lambda_{k}}{\lambda_{1}}\right)^{n-1} \frac{x_{2}}{x_{1}}+\cdots+\alpha_{k}\left(\frac{\lambda_{k}}{\lambda_{1}}\right)^{n-(k-1)} \frac{x_{2}}{x_{1}}=0
\end{gathered}
$$

Notice that we have a system of equations, thus it suffices to show that (2.5) has a solution.

Now, since $\left(\frac{\lambda_{j}}{\lambda_{i}}\right) \neq 0$, for $j=2,3, \ldots, k$, we can divide each equation from the second equation 
by $\left(\frac{\lambda_{k}}{\lambda_{1}}\right)^{n-(k-2)}$ to get the following equivalent system:

$$
\left(\begin{array}{ccccc}
\left(\frac{\lambda_{2}}{\lambda_{1}}\right)^{k-1} \frac{x_{2}}{x_{1}} & \left(\frac{\lambda_{3}}{\lambda_{1}}\right)^{k-2} \frac{x_{2}}{x_{1}} & \ldots & \left(\frac{\lambda_{2}}{\lambda_{1}}\right)^{2} \frac{x_{2}}{x_{1}} & \left(\frac{\lambda_{2}}{\lambda_{1}}\right) \frac{1}{x_{1}} \\
\left(\frac{\lambda_{3}}{\lambda_{1}}\right)^{k-1} \frac{x_{3}}{x_{1}} & \left(\frac{\lambda_{3}}{\lambda_{1}}\right)^{k-2} \frac{x_{3}}{x_{1}} & \ldots & \left(\frac{\lambda_{3}}{\lambda_{1}}\right)^{2} \frac{x_{3}}{x_{1}} & \left(\frac{\lambda_{3}}{\lambda_{1}}\right) \frac{x_{3}}{x_{1}} \\
\vdots & \vdots & \ldots & \vdots \\
\left(\frac{\lambda_{k}}{\lambda_{1}}\right)^{k-1} \frac{x_{k}}{x_{1}} & \left(\frac{\lambda_{k}}{\lambda_{1}}\right)^{k-2} \frac{x_{k}}{x_{1}} & & \left(\frac{\lambda_{k}}{\lambda_{1}}\right)^{2} \frac{x_{k}}{x_{1}} & \left(\frac{\lambda_{k}}{\lambda_{1}}\right) \frac{x_{k}}{x_{1}}
\end{array}\right)\left(\begin{array}{c}
\alpha_{1} \\
\alpha_{2} \\
\alpha_{3} \\
\vdots \\
\alpha_{k}
\end{array}\right)=\left(\begin{array}{c}
1 \\
0 \\
0 \\
\vdots \\
0
\end{array}\right)
$$

To this very end, it remains to show that the determinant of the matrix

is different from 0 since we have a non-homogeneous system.

$$
B=\left(\begin{array}{cccccc}
\left(\frac{\lambda_{2}}{\lambda_{1}}\right)^{k-1} \frac{x_{2}}{x_{1}} & \left(\frac{\lambda_{3}}{\lambda_{1}}\right)^{k-2} \frac{x_{2}}{x_{1}} & \cdots & \left(\frac{\lambda_{2}}{\lambda_{1}}\right)^{2} \frac{x_{2}}{x_{1}} & \left(\frac{\lambda_{2}}{\lambda_{1}}\right) \frac{x_{2}}{x_{1}} \\
\left(\frac{\lambda_{3}}{\lambda_{1}}\right)^{k-1} \frac{x_{3}}{x_{1}} & \left(\frac{\lambda_{3}}{\lambda_{1}}\right)^{k-2} \frac{x_{3}}{x_{1}} & \ldots & \left(\frac{\lambda_{3}}{\lambda_{1}}\right)^{2} \frac{x_{3}}{x_{1}} & \left(\frac{\lambda_{3}}{\lambda_{1}}\right) \frac{x_{3}}{x_{1}} \\
\vdots & \vdots & \cdots & \vdots & \vdots \\
\left(\frac{\lambda_{k}}{\lambda_{1}}\right)^{k-1} \frac{x_{k}}{x_{1}} & \left(\frac{\lambda_{k}}{\lambda_{1}}\right)^{k-2} \frac{x_{k}}{x_{1}} & & \left(\frac{\lambda_{k}}{\lambda_{1}}\right)^{2} \frac{x_{k}}{x_{1}} & \left(\frac{\lambda_{k}}{\lambda_{1}}\right) \frac{x_{k}}{x_{1}}
\end{array}\right)
$$

We observe that each row of the matrix $B$ is a geometric progression, hence $B$ is a $k \times k$ VanderMonde matrix, hence $|B|$ can be computed from the well known formula for VanderMonde matrix and we get,

$$
|B|=\frac{1}{\lambda_{1}^{\frac{k(k-1)}{2}}} \frac{x_{2} x_{3} \ldots x_{k}}{x_{1}^{k-1}} \prod_{1 \leq i \leq j \leq k}\left(\lambda_{j}-\lambda_{i}\right),
$$

which is clearly $\neq 0$ since the quantity outside the product is non-zero and the product is nonzero as the $\lambda_{j}$ 's are all distinct. This ends the proof of the theorem.

The next two theorems give further results on the convergence of orbits. We take the set eigenvalues between 0 and 1 and obtain some results on strong and weak convergence.
Theorem 2.5. Let $T=\left(\begin{array}{ccccc}r_{1} & 0 & 0 & \ldots & \\ 0 & r_{2} & 0 & \ldots & \\ 0 & 0 & r_{3} & 0 & \ldots \\ \vdots & \vdots & \vdots & \ddots & \vdots\end{array}\right)$, $T: \ell^{2} \rightarrow \ell^{2}$ be such that $r_{i} \neq r_{j}$ for $i \neq j$, and the $r_{i}$ 's are real numbers between 0 and 1 .

Let $x=\left(x_{1}, x_{2}, x_{3}, \cdots\right) \in \ell^{2}$ where $x_{i} \neq 0$ for all $i$. Suppose further that $\sup _{i} r_{i}=1$ and $\inf _{i} r_{i}=0$.

(a) If $\sup r_{j}=r_{j_{0}}=1$ for some $j_{0}$ then, $\lim _{n \rightarrow \infty} \frac{T^{n_{x}}}{\left\|T^{n} x\right\|}=e_{j_{0}} \operatorname{sign}\left(x_{j_{0}}\right) \quad$ where $\quad e_{j_{0}}=$ $(0,0,0, \ldots, 1,0,0, \ldots 0)$, one on the $j_{0}$ - position and zero elsewhere.

(b) If the supremum is not attained then $\frac{T^{n} x}{\left\|T^{n} x\right\|} \rightarrow 0$ weakly.

Proof. (a) Let $x=\left(x_{1}, x_{2}, x_{3}, x_{j_{0}}, x_{j_{0+1}} \ldots\right)$, where $x_{j_{0}} \neq 0$ and $r_{j_{0}}=1$. We have So,

$$
T^{n} x=\left(r_{1}^{n} x_{1}, r_{2}^{n} x_{2}, r_{3}^{n} x_{3}, \ldots, r_{j_{0-1}}^{n} x_{j_{0}}, r_{j_{0}}^{n} x_{j_{0}}, r_{j_{0+1}}^{n} x_{j_{0+1}}, \cdots\right)
$$


$\left\|T^{n} x-\left(0,0,0, x_{j_{0}}, 0,0,0, \ldots\right)\right\|_{\rho^{2}}=$

$=\left\|\left(r_{1}^{n} x_{1}, r_{2}^{n} x_{2}, r_{3}^{n} x_{3}, \ldots, r_{j_{0-1}}^{n} x_{j_{0-1}}, r_{j_{0}}^{n} x_{j_{0}}, r_{j_{0+1}}^{n} x_{j_{0+1},}, \ldots\right)-\left(0,0,0, x_{j_{0}}, 0,0,0, \ldots\right)\right\|$

$=\left\|\left(r_{1}^{n} x_{1}, r_{2}^{n} x_{2}, r_{3}^{n} x_{3}, \ldots, r_{j_{0-1}}^{n} x_{j_{0-1}}, 0, r_{j_{0+1}}^{n} x_{j_{0+1}} \ldots\right)\right\|$

$\sqrt{\left(r_{1}^{2 n} x_{1}^{2}+r_{2}^{2 n} x_{2}^{2}+r_{3}^{2 n} x_{3}^{2}+\cdots+r_{j_{0-1}}^{n} x_{j_{0-1}}+0+r_{j_{0+1}}^{n} x_{j_{0+1}}+\cdots\right)}$

But $x=\left(x_{1}, x_{2}, x_{3}, \cdots\right) \in \ell^{2}$, so $\exists N \in \mathbb{N}$ such that $\sum_{k=N+1}^{\infty}\left|x_{k}\right|^{2}<\epsilon \forall \epsilon>0$. Since

$0<r_{j}<1, \forall j \neq j_{0}$, we have that,

Notice that,

$$
\sum_{k=N+1}^{\infty}\left|x_{k}\right|^{2}\left|r_{k}^{n}\right|^{2}<\epsilon
$$

$$
\begin{aligned}
& \sqrt{r_{1}^{2 n} x_{1}^{2}+r_{2}^{2 n} x_{2}^{2}+r_{3}^{2 n} x_{3}^{2}+\cdots+r_{j_{0-1}}^{n} x_{j_{0-1}}+0+r_{j_{0+1}}^{n} x_{j_{0+1}}+\cdots}= \\
& =\sqrt{\sum_{k=1}^{\infty}\left|x_{k}\right|^{2}\left|r_{k}^{n}\right|^{2}+\sum_{k=N+1}^{\infty}\left|x_{k}\right|^{2}\left|r_{k}^{n}\right|^{2}} \\
& =\sqrt{I+I I}
\end{aligned}
$$

Where $I=\sum_{k=1}^{\infty}\left|x_{k}\right|^{2}\left|r_{k}^{n}\right|^{2}$ and $I I=\sum_{k=N+1}^{\infty}\left|x_{k}\right|^{2}\left|r_{k}^{n}\right|^{2}$.

But $I I<\epsilon_{1}$ by the arguments above. Thus, it remains to show that $I<\epsilon_{2}$ too.

Indeed $I<\epsilon_{2}$ since there are only finitely many terms and the $r_{j}$ 's are such that $0<r_{j}<1, \forall j \neq$ $j_{0}$. Hence we have shown that $T^{n} x \rightarrow\left(0,0,0, \ldots, x_{j_{0}}, 0,0,0, \ldots\right)$. Thus, $\frac{T^{n} x}{\left\|T^{n} x\right\|} \rightarrow e_{j_{0}} \operatorname{sign}\left(x_{j_{0}}\right)$, and this finishes the proof of (a).

(b) Let $\frac{T^{n} x}{\left\|T^{n} x\right\|}=\left(a_{1}, a_{2}, a_{3}, \ldots, a_{j-1}, a_{j}, a_{j+1}, \ldots\right)$. Now, if the supremum is not attained, then for any particular $j$, there exist $r_{j}$ such that $r_{j}>r_{j_{0}}$, for some $j>j_{0}$. we have,

But by assumption, $\frac{r_{j}^{n} x_{j}}{\left\|T^{n} x\right\|} \rightarrow a_{j}$ and $\frac{r_{j_{0}}^{n} x_{j_{0}}}{\left\|T^{n} x\right\|} \rightarrow a_{j_{0}}$

$$
\frac{T^{n} x}{\left\|T^{n} x\right\|}=\left(\ldots, \frac{r_{j}^{n} x_{j}}{\left\|T^{n} x\right\|}, \ldots, \frac{r_{j_{0-1}}^{n} x_{j_{0-1}}}{\left\|T^{n} x\right\|}, \frac{r_{j_{0}}^{n} x_{j_{0}}}{\left\|T^{n} x\right\|}, \frac{r_{j_{0+1}}^{n} x_{j_{0+1}}}{\left\|T^{n} x\right\|}, \ldots\right) .
$$

$\Rightarrow \frac{a_{j_{0}}}{a_{j}}=\lim _{j \rightarrow \infty} \frac{r_{j_{0}}^{n} x_{j_{0}}}{r_{j}^{n} x_{j}}=0$ Since $r_{j}>r_{j_{0}}$, for some $j>j_{0}$.

Further, we have that $\frac{T^{n} x}{\left\|T^{n} x\right\|}=1$, so the sequence $\left(\frac{T^{n} x}{\left\|T^{n} x\right\|}\right)$ is bounded.

Thus, we have shown that the coordinates of $\frac{T^{n} x}{\left\|T^{n} x\right\|} \rightarrow 0$ pointwise and the sequence is bounded.

Hence, $\frac{T^{n} x}{\left\|T^{n} x\right\|} \rightarrow 0$ weakly, this completes the proof of (b).

Corollary 2.6. Let the operator $T$ and the vector $x$ be defined as in the previous theorem. Suppose that $\sup _{i} r_{i}=1$ but 1 is not attained. Then $\lim _{n \rightarrow \infty} T^{n} x=0$, in the norm topology for every $x$.

Proof. We want to show that $\left\|T^{n} x-0\right\|=\left\|T^{n} x\right\| \rightarrow 0$ as $n \rightarrow \infty$. As before, $x=\left(x_{1}, x_{2}, x_{3}, \ldots\right) \in l_{2}$, so $\exists N \in \mathbb{N}$ such that $\sum_{k=N+1}^{\infty}\left|x_{k}\right|^{2}<\epsilon \forall \epsilon>0$.

Now we have, 


$$
\begin{gathered}
\left\|T^{n} x\right\|=\sqrt{r_{1}^{2 n} x_{1}^{2}+r_{2}^{2 n} x_{2}^{2}+r_{3}^{2 n} x_{3}^{2}+\cdots+r_{j}^{n} x_{j}+r_{j+1}^{n} x_{j+1}+\cdots} \\
=\sqrt{r_{1}^{2 n} x_{1}^{2}+r_{2}^{2 n} x_{2}^{2}+\ldots+r_{N}^{n} x_{N}+r_{N+1}^{n} x_{N+1}+r_{N+2}^{n} x_{N+2}+\cdots} \\
=\sqrt{A+B}
\end{gathered}
$$

Where: $A=r_{1}^{2 n} x_{1}^{2}+r_{2}^{2 n} x_{2}^{2}+\ldots+r_{N}^{n} x_{N}$ and $B=r_{N+1}^{n} x_{N+1}+r_{N+2}^{n} x_{N+2}+\cdots$

Notice $A \in \epsilon_{1}$ by the remark above $\left(x \in \ell^{2}\right)$ and the fact that $0<r_{j}<1, \forall j$. But also $B<\epsilon_{2}$ since there are only finitely many terms and the $r_{j}$ 's are such that $0<r_{j}<1, \forall j$.

$\Rightarrow \sqrt{A+B}<\sqrt{\epsilon_{1}+\epsilon_{2}}<\epsilon_{3}$, and the theorem is proved.

We end this paper with an example from normed vector spaces that utilizes Theorem 2.4.

Example 2.7 Let $X$ be a separable normed vector space. Suppose that $\left\{x_{1}, x_{2}, x_{3}, \ldots.\right\}$ is a set of linearly independent elements in a normed linear space $X$ with the property that each $x \in X$ can be well approximated by elements in the linear space spanned by the $x_{n}$ 's. That is, for each $x \in X$ and each $>0$, there exist scalars $\alpha_{i}, i=1,2,3, \ldots, n$ such that for $y=$ $\sum_{i=1}^{n} \alpha_{i} x_{i}$, we have $\|x-y\|<\varepsilon$. Then by defining the linear subspaces $M_{n}$ of $X$ by $M_{n}=\operatorname{span}\left\{x_{1}, x_{2}, x_{3}, \ldots x_{n}\right\}$, we get the following:

$\operatorname{dim} M_{n}=n$ and $M_{n} \subset M_{n+1}$ for all $n$ and $M_{n} X=\overline{\mathrm{U}_{n=1}^{\infty} M_{n}}$.

\section{Conclusions}

The findings of this study show that, the basis elements of the space $\ell^{2}$ can be generated by using the linear combinations of the elements of the orbit of the diagonal operator defined on $\ell^{2}$. Furthermore, the rate of convergence of the orbit to a particular basis element depends on the number of elements of the orbit: the more the elements of the orbit, the faster the convergence.

\section{References}

Bögli S 2017 Convergence of sequences of linear operators and their spectra. Integral Equat. Oper. Theory 88(4): 559-599.

Deutsch F and Hundal H 2010 Slow convergence of sequences of linear operators II: Arbitrarily slow convergence. J. Approx. Theory 162(9): 1717-1738.

Enflo E 1987 On the invariant subspace problem for Banach spaces. Acta Math. 158(1): 213-313.

Mančevska S and Orovčanec M 2007 Orbits tending to infinity under sequences of operators on Hilbert spaces. Filomat 21(2): 161-171.

Müller V and Vršovský J 2009 Orbits of linear operators tending to infinity. Rocky Mt. J. Math. 39(1): 219-230.

Read CJ 1985 A solution to the invariant subspace problem on the space $\ell^{1}$. Bull. London Math. Soc. 17(4): 305-317.

Szász O 1916 Übe die Approximation stetiger Funktionen durch lineare Aggregate von Potenzen. Math. Ann. 77(4): 482-496. 